\title{
Perfil da saúde vocal de cantores amadores de igreja evangélica
}

\section{Vocal health profile of amateur singers from an evangelical church}

\author{
Thaise Marcela Mota Barreto ${ }^{1}$, Geová Oliveira de Amorim¹, Euclides Mauricio Trindade Filho², \\ Célia Akemi Kanashiro ${ }^{3}$
}

\begin{abstract}
RESUMO
Objetivo: Investigar o perfil de saúde vocal de cantores amadores de louvores evangélicos com relação a queixas, hábitos e dificuldades vocais vivenciados durante a prática do canto em indivíduos dos gêneros masculino e feminino. Métodos: Participaram 55 sujeitos, com idade entre 18 e 50 anos, os quais responderam a um questionário auto-aplicável sobre identificação de hábitos vocais e possíveis queixas de voz falada e cantada. Resultados: Os cantores religiosos evangélicos amadores relataram queixas relacionadas à voz, tais como rouquidão constante $(43,6 \%)$, pigarro constante $(43,6 \%)$, falhas na voz $(34,5 \%)$, perda de voz $(18,1 \%)$, garganta seca $(18,1 \%)$, voz fraca $(14,5 \%)$ e dor no pescoço e na nuca $(12,7 \%)$. Durante a atividade de canto, as queixas mais reportadas foram as de dificuldades de alcançar notas agudas (45,4\%), rouquidão (30,9\%) e falhas na voz (29\%). Os hábitos vocais expostos pelos cantores foram os relativos a falar muito (63,6\%), ingerir gelado habitualmente em excesso (43,6\%), falar alto (40\%) e gritar com frequência (20\%). Com relação à variável gênero, foram observadas diferenças significativas entre os grupos, relacionadas às variáveis falhas na voz, consumo de gelados e falar alto. Conclusão: Os cantores religiosos amadores, de ambos os gêneros, apresentam percentual expressivo de queixas e hábitos vocais que podem estar associados à falta de informações sobre os hábitos saudáveis de produção vocal e que podem contribuir para o desenvolvimento de alterações laríngeas e disfonia.
\end{abstract}

Descritores: Voz; Disfonia: Qualidade da voz; Distúrbios da voz; Questionários

\section{INTRODUÇÃO}

A voz humana é uma poderosa ferramenta de comunicação e constitui uma das mais fortes extensões da personalidade de um indivíduo. A modulação, a intensidade, a projeção e a qualidade vocal transmitem o estado de humor do falante, interferindo decisivamente na eficácia da comunicação ${ }^{(1)}$.

Os mesmos órgãos fonoarticulatórios são utilizados para falar e cantar, porém, a voz cantada exige ajustes vocais diferenciados conforme as exigências da música e cuidados específicos para manter a saúde vocal ${ }^{(2-4)}$. Desta forma, para os cantores, o conhecimento a respeito da inter-relação entre ciência e arte é essencial para o adequado aperfeiçoamento do canto. De forma contrária, este desconhecimento pode in-

Trabalho realizado no Curso de Fonoaudiologia, Universidade Estadual de Ciências da Saúde de Alagoas - UNCISAL - Maceió (AL), Brasil.

(1) Faculdade de Fonoaudiologia, Universidade Estadual de Ciências da Saúde de Alagoas - UNCISAL - Maceió (AL), Brasil.

(2) Departamento de Ciências Fisiológicas, Universidade Estadual de Ciências da Saúde de Alagoas - UNCISAL - Maceió (AL), Brasil.

(3) Universidade Estadual de Ciências da Saúde de Alagoas - UNCISAL Maceió (AL).

Endereço para correspondência: Thaise Marcela Mota Barreto. R. Prof. Valdice Andrade, 162, Inácio Barbosa, Aracaju (SE), Brasil, CEP: 49040-530.

E-mail: thaisefga@hotmail.com

Recebido em: 17/8/2009; Aceito em: 10/11/2010 terferir no bom desempenho de suas atividades, na qualidade e na produtividade da $\mathrm{voz}^{(5,6)}$. A necessidade de orientações e acompanhamento fonoaudiológico é fundamental em todas as modalidades de canto, pois possibilita estratégias saudáveis de produção vocal aos cantores.

O canto religioso é praticado desde as primeiras civilizações humanas. Historiadores encontraram composições religiosas na cultura suméria, caracterizadas pelos hinos, ladainhas e lamentações. Entre os antigos egípcios, assírios, caldeus e hebreus o canto era praticado em cerimônias religiosas e para animar as tropas nas batalhas ${ }^{(7)}$.

Hoje, o canto religioso está presente nas igrejas como forma de adoração, oração e súplica. Os cantores religiosos, principalmente os amadores, estão mais preocupados em transmitir mensagens por meio da música e muitas vezes não se importam com a qualidade do canto. A grande maioria pratica o canto durante vários anos sem nunca ter estudado canto ou fazer uso de alguma técnica vocal ${ }^{(4)}$.

Além do despreparo evidenciado nos cantores religiosos, há também a presença de aspectos de religiosidade e espiritualidade que interferem no comportamento vocal ${ }^{(8-10)}$.

Em estudo realizado com cantores religiosos pertencentes às denominações católica e evangélica ${ }^{(8)}$, observou-se que os cantores da Igreja Evangélica da Assembléia de Deus, comparados aos cantores da Igreja Católica, apresentavam maior 
frequência de canto por semana, expressivo número de queixas vocais, bem como grande exaltação dos fiéis, cantando e participando do culto.

Vale ressaltar que o louvor a Deus é visto pelos fiéis como imprescindível na prática religiosa e essa importância pode ser observada em diversas passagens bíblicas, tais como: "Cantai louvores a Deus, cantai louvores" - Salmos 47:6a; "Estejam na sua garganta os altos louvores de Deus" - Salmos 149:6a; "E estavam sempre no templo, louvando a Deus" - Lucas 24:53; "Falando entre vós com salmos, e hinos, e cânticos espirituais, cantando e salmodiando ao Senhor no vosso coração" - Efésios 5:19. Por meio desse ato os fiéis expressam seus sentimentos e emoções, exaltam e adoram a Deus e fazem suas petições e agradecimentos $^{(10)}$.

Diante deste cenário, o objetivo desse estudo foi investigar o perfil de saúde vocal dos cantores amadores de louvores evangélicos quanto a queixas, hábitos e dificuldades vocais vivenciados por esses indivíduos. A partir dos dados obtidos será possível desenvolver estratégias para promoção da saúde vocal e prevenção de doenças relacionadas ao mau uso da voz cantada junto a essa população.

\section{MÉTODOS}

Esta pesquisa, de caráter descritivo, foi avaliada e aprovada pelo Comitê de Ética em Pesquisa da Universidade Estadual de Ciências da Saúde de Alagoas - UNCISAL, sob protocolo $\mathrm{n}^{\circ} 972$.

Participaram inicialmente deste estudo 64 sujeitos, dos quais foram excluídos nove participantes em virtude de não terem preenchido corretamente o protocolo, totalizando um número de 55 sujeitos, cantores amadores frequentadores de diversas Igrejas Evangélicas da Assembléia de Deus da cidade de Maceió, sendo 29 do gênero masculino e 26 do gênero feminino, com faixa etária entre 18 e 55 anos e com tempo de prática de canto na igreja maior ou igual a 6 meses. A amostra deveria ser composta exclusivamente por solistas, porém, em função da dinâmica da igreja, não foi possível encontrar sujeitos com essa característica, pois os indivíduos que cantam solo também participam de grupos de louvor.

O tamanho da amostra foi definido levando-se em consideração a variável "sintomas vocais" de um estudo destinado a cantores de corais, tendo sido estimada uma proporção da população de $79,3 \%{ }^{(11)}$, com precisão absoluta de $10 \%$ e o nível de significância de $5 \%{ }^{(12)}$.

Foram excluídos do estudo os sujeitos que estavam em atendimento fonoaudiológico, os que já realizaram terapia fonoaudiológica relacionada à voz e aqueles que realizaram ou realizam aula de técnica vocal com professor de canto.

Os sujeitos selecionados foram esclarecidos sobre a pesquisa e seus aspectos éticos, e assinaram o Termo de Consentimento Livre e Esclarecido.

Com base na literatura ${ }^{(3,13)}$ empregou-se um questionário auto-aplicável, estruturado e adaptado (Anexo 1) dirigido aos sujeitos da pesquisa, cujo objetivo foi investigar o perfil de saúde vocal, considerando-se as variáveis dependentes (saúde vocal dos cantores religiosos e dados relativos a possíveis queixas, hábitos e dificuldades vocais) e independentes: da- dos de identificação (gênero, idade, escolaridade); dados de hábitos vocais (tempo de prática do canto, classificação vocal, aquecimento vocal e hidratação. O questionário foi aplicado pelo próprio avaliador a cada participante da pesquisa de forma individual para que possíveis dúvidas fossem esclarecidas no momento da marcação das respostas.

Os dados desta pesquisa foram tabulados em planilha Excel e processados pelo aplicativo Statistical Package for Social Sciences (SPSS, versão 16.0), analisados e apresentados em tabelas, aplicando-se a eles a estatística descritiva com os dados apresentados em forma de frequência. Considerou-se significativo o valor de $\mathrm{p} \leq 0,05$.

\section{RESULTADOS}

A amostra foi composta por 55 cantores amadores de louvores evangélicos das Igrejas Evangélicas da Assembléia de Deus da cidade de Maceió. A distribuição por gênero neste grupo foi de 29 indivíduos (52,7\%) do gênero masculino e 26 (47,2\%) do gênero feminino, com média de idade de 25 anos. A escolaridade predominante na amostra foi de $41,8 \%$ de cantores com ensino médio completo e $30,9 \%$ com ensino superior incompleto.

Os dados obtidos pela utilização do questionário autoaplicável (Anexo 1) revelaram que a maior parte dos sujeitos da pesquisa canta há mais de cinco anos, possuem classificação vocal, não fazem aquecimento vocal e relataram perceber mudanças negativas na voz após iniciar a atividade do canto. Quanto à hidratação, houve uma frequência relativamente homogênea quanto ao número de copos de água ingeridos diariamente, apresentando uma tendência acima de quatro copos diários (Tabela 1) .

Tabela 1. Distribuição numérica e percentual quanto à caracterização dos sujeitos e seus hábitos

\begin{tabular}{|c|c|c|c|}
\hline \multirow[t]{2}{*}{ Variáveis } & & \multicolumn{2}{|c|}{$\begin{array}{c}\text { Cantores evangélicos } \\
\text { amadores }\end{array}$} \\
\hline & & $\mathrm{n}$ & $\%$ \\
\hline \multirow{5}{*}{ Tempo de canto (anos) } & 1 & 1 & 1,8 \\
\hline & 2 & 4 & 7,2 \\
\hline & 3 & 3 & 5,4 \\
\hline & 4 & 1 & 1,8 \\
\hline & Mais de 5 & 41 & 83,6 \\
\hline \multirow{7}{*}{ Classificação vocal } & Não tem & 5 & 9 \\
\hline & Soprano & 9 & 16,3 \\
\hline & Mezzo & 5 & 9 \\
\hline & Contralto & 9 & 16,3 \\
\hline & Baixo & 1 & 1,8 \\
\hline & Tenor & 16 & 29 \\
\hline & Barítono & 8 & 14,5 \\
\hline \multirow{2}{*}{ Aquecimento vocal } & Sim & 18 & 32,8 \\
\hline & Não & 37 & 67,2 \\
\hline \multirow{2}{*}{$\begin{array}{l}\text { Mudança negativa na voz } \\
\text { após início da atividade de } \\
\text { canto na igreja }\end{array}$} & Sim & 45 & 81,8 \\
\hline & Não & 10 & 18,2 \\
\hline \multirow{4}{*}{$\begin{array}{l}\text { Ingestão de água } \\
\text { (copos/dia) }\end{array}$} & 1 a 2 & 9 & 16,3 \\
\hline & 3 a 4 & 13 & 23,6 \\
\hline & 5 a 6 & 18 & 32,7 \\
\hline & Mais de 7 & 14 & 25,4 \\
\hline
\end{tabular}


Os achados relativos a queixas vocais apresentadas pelos cantores evangélicos amadores indicam que as reclamações de maior representatividade foram relativas à rouquidão, pigarro constante, falhas na voz, perda de voz, garganta seca e voz fraca (Tabela 2). É interessante notar que a queixa referente a "falhas na voz" foi significativamente mais frequente nas mulheres do que nos homens. As queixas de menor frequência foram as relativas a dores na nuca/pescoço, sensação de corpo estranho na garganta, dor na garganta ao falar, cansaço ao falar, dor ao engolir, tosse constante, ardência ao falar e ar na voz.

Dentre os problemas vivenciados pelos cantores durante a atividade do canto, os mais frequentes foram dificuldade de alcançar notas agudas, rouquidão, falhas na voz, dificuldade em realizar transição de uma região média para aguda e desconforto na garganta; enquanto que os menos comuns foram dor na garganta, ar na voz e perda de voz (Tabela 3). Novamente verificou-se que o item "falhas na voz" foi maior nas mulheres do que nos homens $(\mathrm{p}=0,0001)$.

Os hábitos vocais inadequados mais adotados pelos can- tores evangélicos amadores foram falar muito, ingerir gelado em excesso, falar alto e/ou gritar com constância (Tabela 4). Houve diferença significativa entre mulheres e homens, mostrando que as mulheres usam mais gelado e também possuem o hábito de falar mais alto.

\section{DISCUSSÃO}

Existem poucos estudos científicos detalhando o comportamento vocal de cantores religiosos amadores que participam de grupo de louvor nas igrejas evangélicas do país. A maioria dos estudos encontrados é direcionada a cantores de coral.

De acordo com os aspectos de identificação pesquisados, dos 55 cantores avaliados, $52,7 \%$ são do gênero masculino, diferindo da maior parte de estudos relacionados ao canto, onde a maioria é composta por indivíduos do gênero feminino ${ }^{(4,8,10)}$. Esse achado pode revelar o maior movimento dos homens em direção à religiosidade, envolvendo-se em atividades mais dinâmicas nas igrejas como, por exemplo, a atividade de canto.

Tabela 2. Distribuição numérica e percentual das queixas vocais apresentadas por cantores evangélicos amadores do gênero masculino e feminino

\begin{tabular}{|c|c|c|c|c|c|c|c|}
\hline \multirow{2}{*}{ Queixas } & \multicolumn{2}{|c|}{ Total } & \multicolumn{2}{|c|}{ Homens } & \multicolumn{2}{|c|}{ Mulheres } & \multirow{2}{*}{ Valor de $p$} \\
\hline & $\mathrm{n}$ & $\%$ & $\mathrm{n}$ & $\%$ & $\mathrm{n}$ & $\%$ & \\
\hline Rouquidão & 24 & 43,6 & 14 & 48,7 & 10 & 38,4 & 0,1969 \\
\hline Pigarro constante & 24 & 43,6 & 14 & 48,7 & 10 & 38,4 & 0,1969 \\
\hline Falhas na voz & 19 & 34,5 & 7 & 24,1 & 12 & 46,1 & $0,0018^{*}$ \\
\hline Perda de voz & 10 & 18,1 & 6 & 20,6 & 4 & 15,3 & 0,4562 \\
\hline Garganta seca & 10 & 18,1 & 4 & 13,7 & 6 & 23,07 & 0,0973 \\
\hline Voz fraca & 8 & 14,5 & 5 & 17,2 & 3 & 11,5 & 0,3078 \\
\hline Dor no pescoço/nuca & 7 & 12,7 & 3 & 10,3 & 4 & 15,3 & 0,3921 \\
\hline Sensação de corpo estranho na garganta & 6 & 10,9 & 3 & 10,3 & 3 & 11,5 & 1,000 \\
\hline Dor ao engolir & 5 & 9,09 & 2 & 6,89 & 3 & 11,5 & 0,3103 \\
\hline Dor na garganta ao falar & 5 & 9,09 & 2 & 6,89 & 3 & 11,5 & 0,3103 \\
\hline Cansaço ao falar & 5 & 9,09 & 2 & 6,89 & 3 & 11,5 & 0,3103 \\
\hline Tosse constante & 4 & 7,27 & 3 & 10,3 & 1 & 3,84 & 0,0851 \\
\hline Ar na voz & 2 & 3,63 & 1 & 3,44 & 1 & 3,84 & 0,5507 \\
\hline Ardência ao falar & 2 & 3,63 & 1 & 3,44 & 1 & 3,84 & 0,5507 \\
\hline
\end{tabular}

*Valores significativos $(p \leq 0,05)$ - Teste Qui-quadrado

Tabela 3. Distribuição numérica e percentual dos problemas vivenciados durante a atividade do canto por cantores evangélicos amadores do gênero masculino e feminino

\begin{tabular}{|c|c|c|c|c|c|c|c|}
\hline \multirow{2}{*}{ Problemas vivenciados } & \multicolumn{2}{|c|}{ Total } & \multicolumn{2}{|c|}{ Homens } & \multicolumn{2}{|c|}{ Mulheres } & \multirow[t]{2}{*}{ Valor de $\mathrm{p}$} \\
\hline & $\mathrm{n}$ & $\%$ & $\mathrm{n}$ & $\%$ & $\mathrm{n}$ & $\%$ & \\
\hline Dificuldade em alcançar notas agudas & 25 & 45,4 & 14 & 48,2 & 11 & 42,3 & 0,4755 \\
\hline Rouquidão & 17 & 30,9 & 10 & 34,4 & 7 & 26,9 & 0,2790 \\
\hline Falhas na voz & 16 & 29 & 4 & 13,7 & 12 & 46,1 & $0,0001^{*}$ \\
\hline $\begin{array}{l}\text { Dificuldade em realizar transição de uma região média } \\
\text { para aguda }\end{array}$ & 13 & 23,6 & 7 & 24,1 & 6 & 23 & 0,9686 \\
\hline Desconforto na garganta & 11 & 20 & 8 & 27,5 & 5 & 19,3 & 0,2388 \\
\hline Dor na garganta & 6 & 10,9 & 2 & 6,8 & 4 & 15,3 & 0,0648 \\
\hline Ar na voz & 4 & 7,2 & 2 & 6,8 & 2 & 7,6 & 1,000 \\
\hline Perda de voz (afonia) & 3 & 5,4 & 2 & 6,8 & 1 & 3,8 & 0,4950 \\
\hline
\end{tabular}

* Valores significativos $(p \leq 0,05)$ - Teste qui-quadrado 
Tabela 4. Distribuição numérica e percentual dos hábitos vocais inadequados adotados por cantores evangélicos amadores do gênero masculino e feminino

\begin{tabular}{|c|c|c|c|c|c|c|c|c|}
\hline \multirow[t]{2}{*}{ Abusos vocais } & & \multicolumn{2}{|c|}{ Total } & \multicolumn{2}{|c|}{ Homens } & \multicolumn{2}{|c|}{ Mulheres } & \multirow[t]{2}{*}{ Valor de $p$} \\
\hline & & $\mathrm{n}$ & $\%$ & $\mathrm{n}$ & $\%$ & $\mathrm{n}$ & $\%$ & \\
\hline \multirow[t]{2}{*}{ Fala excessivamente } & Sim & 35 & 63,6 & 17 & 58,6 & 18 & 69,2 & 0,1384 \\
\hline & Não & 20 & 36,4 & 12 & 41,4 & 8 & 30,8 & \\
\hline \multirow[t]{2}{*}{ Faz uso de gelado } & $\operatorname{Sim}$ & 24 & 43,6 & 10 & 34,4 & 14 & 53,8 & $0,010^{*}$ \\
\hline & Não & 31 & 56,4 & 19 & 65,6 & 12 & 46,2 & \\
\hline \multirow[t]{2}{*}{ Fala alto } & Sim & 22 & 40 & 5 & 17,2 & 17 & 65,3 & $0,0001^{*}$ \\
\hline & Não & 33 & 60 & 24 & 82,8 & 9 & 34,7 & \\
\hline \multirow[t]{2}{*}{ Grita } & Sim & 11 & 20 & 6 & 20,6 & 5 & 19,2 & 1,000 \\
\hline & Não & 44 & 80 & 23 & 79,4 & 21 & 80,8 & \\
\hline
\end{tabular}

* Valores significativos $(p \leq 0,05)$ - Teste Qui-quadrado

Os resultados obtidos na análise dos hábitos vocais revelaram que a maioria dos cantores não exercita a prática do aquecimento vocal antes do uso vocal nas atividades de canto (Tabela 1). Sabe-se que o aquecimento vocal tem ação direta na musculatura laríngea, preparando-a para o uso intensivo da $\operatorname{voz}^{(14)}$. Vários estudos evidenciaram a importância do aquecimento vocal antes dos ensaios e apresentações como mantenedor da voz cantada saudável, melhorando alguns parâmetros vocais e reduzindo possíveis alterações vocais ${ }^{(2,15-17)}$.

Com relação à auto-percepção vocal dos cantores, podese inferir que os resultados encontrados sejam consequência da falta de conhecimentos relativos ao uso da voz e da nãorealização de exercícios que preparem o trato vocal para o uso da mesma, bem como hábitos vocais e fatores orgânicos associados à produção de uma qualidade vocal saudável.

Atualmente, estudos ${ }^{(18,19)}$ vêm sendo desenvolvidos procurando correlacionar os efeitos da hidratação na regulação da vibração das pregas vocais. Sabe-se que a desidratação leva ao aumento da viscosidade nas pregas vocais, que gera alterações na qualidade vocal, inadequação da vibração da prega vocal e pigarro ${ }^{(20-22)}$. É interessante ressaltar alguns fatores que podem comprometer a hidratação, tais como a ingestão insuficiente de água, baixos níveis de umidade do ar e a ação de medicamentos que promovem o ressecamento da mucosa das pregas vocais ${ }^{(23)}$. Neste estudo, os valores referidos pela maioria dos cantores para ingestão de água constituem bons níveis de hidratação, conforme dados da literatura ${ }^{(24)}$.

Em termos de queixas vocais, os indivíduos relataram mais de uma queixa vocal, porém as frequências foram variáveis (Tabela 2). Provavelmente, as queixas de rouquidão e pigarro constante, relatadas por um grande número de cantores, podem ser decorrentes do uso intensivo da voz. Em outro estudo, realizado com cantores de coros evangélicos amadores, foi verificado que a grande maioria pratica o canto durante vários anos, sem nunca estudarem canto ou fazerem uso de alguma técnica vocal ${ }^{(4)}$. Além do despreparo evidenciado nos cantores religiosos, há também a presença de aspectos de religiosidade e espiritualidade que interferem no comportamento vocal desses indivíduos, levando-os a praticarem seus atos religiosos quase sempre em intensidade vocal elevada, o que inclui o ato de cantar ${ }^{(8-10)}$; porém, este estudo não deixa claro aspectos importantes do perfil do estilo vocal dos sujeitos do estudo.
As queixas relativas às falhas, perda da voz (momentos de afonia) e garganta seca possivelmente podem ser decorrentes da combinação da abertura frequente da cavidade oral e do uso prolongado da voz durante a utilização da voz cantada, embora o conceito de uso prolongado de voz ainda não seja claro na literatura científica. Estima-se que durante um dia de trabalho as pregas vocais de um profissional da voz, terão efetuado aproximadamente três milhões de ciclos vibratórios o que, sob o ponto de vista biomecânico, pode ser considerado uma sobrecarga às pregas vocais ${ }^{(19)}$. A sobrecarga vocal é definida como uma combinação entre o tempo de uso da voz e fatores que afetam sua produção, como as condições acústicas do ambiente de trabalho e as exigências de fala e canto ${ }^{(25)}$. A literatura reporta aos sintomas de voz fraca, cansaço vocal, falhas na voz, dores na garganta e rouquidão em indivíduos com uso intensivo de voz como indicativos de sobrecarga vocal ${ }^{(18)}$.

Em relação aos problemas vivenciados pelos cantores durante o canto, os resultados obtidos possivelmente estão associados a fatores relacionados ao desequilíbrio da musculatura laríngea (Tabela 3). Sabe-se que o comprimento e a forma do trato vocal são individualmente estabelecidos e determinados pela idade e gênero ${ }^{(26)}$. Verificou-se que as mulheres apresentaram maior frequiência, comparadas aos homens, com relação ao item falhas na voz; corroborando um possível desequilíbrio muscular laríngeo ligado ao gênero bem como os desvios de proporção glótica ${ }^{(20)}$. É necessário conhecer as medidas de tessitura vocal para o canto, pois desrespeitar essas medidas constitui um risco para problemas laríngeos e vocais, tais como: nódulos, espessamentos, dificuldades nas zonas de passagem de registro, rouquidão e falhas na voz ${ }^{(26)}$. Consequentemente, os problemas reportados pelos cantores podem indicar um quadro incipiente de disfonia.

Os hábitos vocais caracterizam-se por comportamentos vocais capazes de gerar traumas na mucosa laríngea devido ao movimento brusco em oposição às pregas vocais ${ }^{(27)}$ (Tabela 4). Neste trabalho, os hábitos vocais relatados pelos cantores, baseados nas suas percepções, demonstraram que as mulheres apresentam os hábitos de ingerir gelado e falar alto com maior intensidade, comparativamente aos homens. Evidências clínicas mostram que a intensificação dos hábitos vocais encontrados, pode gerar prejuízos às funções laríngea e vocal desses indivíduos ${ }^{(28)}$; portanto, as mulheres desta população, 
especificamente, estariam mais susceptíveis a estes danos.

É importante destacar que estudos baseados em questionários auto-aplicáveis são menos invasivos, menos onerosos, permitem a aplicação em amostras maiores, selecionadas de modo aleatório e não sofrem a interferência do avaliador na aferição, pois, quando o avaliador questiona o sujeito oferecendo-lhe as opções de marcação, as suas características de voz e/ ou a expressão facial podem afetar o preenchimento de dados por parte do sujeito da pesquisa. Além disso, existe o risco de um viés de memória, pois indivíduos mais identificados com as questões da pesquisa podem responder mais atentamente ao questionário ${ }^{(28-30)}$, o que não foi observado nesta investigação; mostrando a fidedignidade das informações coletadas e apresentadas.

Assim, os resultados do presente estudo demonstraram que nesta população estudada, as queixas vocais relatadas podem estar relacionadas à falta de informações quanto ao uso funcional da voz cantada.

As informações obtidas sugerem a importância da presença do fonoaudiólogo na orientação e assessoria vocal de cantores religiosos, com a finalidade de programar medidas preventivas mais eficientes a fim de evitar e/ou diminuir os transtornos laríngeos e vocais dos mesmos.

\section{CONCLUSÃO}

A população de cantores evangélicos estudada apresentou distribuição relativamente homogênea entre indivíduos do gênero masculino e feminino, e a análise da maioria dos quesitos avaliados não apresentou diferenças significativas no tocante às queixas e problemas vocais reportados pelos cantores de ambos os gêneros. Entretanto, diferenças significativas foram observadas entre homens e mulheres em alguns itens pesquisados, como "falhas na voz", "maior consumo de gelados" e "falar mais alto"; em que a frequência foi maior entre as mulheres quando comparadas aos homens. Estas variáveis podem estar sinalizando um provável quadro incipiente de disfonia que pode estar relacionado à falta de informações acerca de hábitos saudáveis de produção vocal, sugerindo a relevância da atuação do profissional fonoaudiólogo concernente à orientação e assessoria dos mesmos.

\begin{abstract}
Purpose: To investigate the vocal health profile of male and female gospel amateur singers regarding vocal complaints, habits and difficulties during singing practice. Methods: Participants were 55 subjects with ages between 18 and 50 years, who answered a self-assessment questionnaire regarding the identification of vocal habits and possible complaints of spoken and sung voice. Results: Amateur gospel singers reported vocal complaints, such as continuous hoarseness (43.6\%), constant throat clearing (43.6\%), voice failure $(34.5 \%)$, voice loss $(18.1 \%)$, dry throat $(18.1 \%)$, weak voice $(14.5 \%)$, and neck and cervix pain (12.7\%). During singing activity, the most reported complaints were difficulties in reaching high notes (45.4\%), hoarseness (30.9\%) and voice failure (29\%). The vocal habits the singers reported regarded excessive talking (63.6\%), immoderate icy beverages intake (43.6\%), loud talking $(40 \%)$, and repeatedly shouting $(20 \%)$. Significant differences were found between genders regarding the variables voice failure, icy beverages intake, and loud talking. Conclusion: Amateur religious singers of both genders showed an expressive percentage of vocal complaints and habits that might be associated to lack of information about healthy vocal habits, which can contribute to the development of laryngeal alterations and voice disorders.
\end{abstract}

Keywords: Voice; Dysphonia; Voice quality; Voice disorders; Questionnaires

\section{REFERÊNCIAS}

1. Titze IR, Lemke J, Montequin D. Populations in the U.S. workforce who rely on voice as a primary tool of trade: a preliminary report. J Voice. 1997;11(3):254-9.

2. Behlau M, Rehder MI. Higiene vocal para o canto coral. Rio de Janeiro: Revinter; 1997.

3. Ribeiro LR, Hanayama EM. Perfil vocal de coralistas amadores. Rev CEFAC. 2005;7(2):252-66.

4. Costa PJBM, Ferreira KI, Camargo ZA, Pinho SMR. Extensão vocal de cantores de coros evangélicos amadores. Rev CEFAC. 2006;8(1):96-106.

5. Murry T, Rosen CA. Vocal education for the professional voice user and singer. Otolaryngol Clin North Am. 2000;33(5):967-82.

6. Ahumanda A. La higiene vocal: una forma de control del fonotrauma. Acta Otorrinolaringol Cir Cabeza Cuello. 2003;31(2 Supl):54-6.

7. Rull MAP. As origens do canto gregoriano. 2001. Disponível em: http:// www.terra.com.br/virtualbooks.

8. Leite GCA, Assumpção R, Campiotto AR, Silva MAA. O canto nas igrejas: o estudo do uso vocal dos coralistas e não-coralistas. Distúrb
Comun. 2004;16(2):229-39.

9. Penteado RZ, Honorato FG, Nascimento JS. Mulher pastora: questões de gênero e condições de uso da voz no meio religioso. Distúrb Comun. 2006;18(3):345-53.

10. Penteado RZ, Silva CR, Pereira PFA. Aspectos de religiosidade na saúde vocal de cantores de grupos de louvor. Rev CEFAC. 2008;10(3):359-68.

11. Rehder MIBC, Behlau MS. Perfil vocal de regentes de coral do estado de São Paulo. Rev CEFAC. 2008;10(2):206-17.

12. Lwanga SK, Lemeshow S. Sample size determination in health studies: a practical manual. Geneva: World Health Organization; 1991.

13. Sapir S, Mathers-Schmidt B, Larson GW. Singers' and non-singers' vocal health, vocal behaviours, and attitudes towards voice and singing: indirect findings from a questionnaire. Eur J Disord Commun. 1996;31(2):193-209.

14. Titze I. Choir warm-ups: how effective are they? J Sing. 2000;56:31-2.

15. Blaylock TR. Effects of systematized vocal warm-up on voices with disorders of various etiologies. J Voice. 1999;13(1):43-50. 
16. Pela SM, Ávila CRB, Behlau M. Análise de parâmetros vocais pré e pós-aquecimento vocal em coralistas. Fono Atual. 2000;(13):26-32.

17. Amir O, Amir N, Michaeli O. Evaluating the influence of warm-up on singing voice quality using acoustic measures. J Voice. 2005;19(2):25260.

18. Chan RW, Tayama N. Biomechanical effects of hydration in vocal fold tissues. Otolaryngol Head Neck Surg. 2002;126(5):528-37.

19. Verdolini K, Titze IR, Fennell A. Dependence of phonatory effort on hydration level. J Speech Hear Res. 1994;37(5):1001-7.

20. Sataloff RT, Spiegel JR. Care of the professional voice. Otolaryngol Clin North Am.1991;24(5):1093-124.

21. Verdolini-Marston K, Sandage M, Titze IR. Effect of hydration treatments on laryngeal nodules and polyps and related voice measures. J Voice. 1994;8(1):30-47.

22. Verdolini-Marston K, Titze IR, Druker DG. Changes in phonation threshold pressure with induced conditions of hydration. J Voice. 1990;4(2):142-51.

23. Thompson AR. Pharmacological agents with effects on voice. Am J Otolaryngol. 1995;16(1):12-8.
24. Pinho SMR. Manual de higiene vocal para profissionais da voz. Carapicuiba: Pró-Fono; 1997.

25. Vilkman E. Voice problems at work: a challenge for occupational safety and health arrangement. Folia Phoniatr Logop. 2000;52(1-3):120-5.

26. Sataloff RT. Anatomia funcional e fisiologia da voz. In: Sataloff RT, Gould WJ, Spiegel JR. Manual prático de fonocirurgia. Rio de Janeiro: Revinter; 2002. p. 63-176.

27. Sakae FA, Sasaki F, Sennes LU, Tsuji DH, Imamura R. Pólipos de pregas vocais e alterações estruturais mínimas: lesões associadas? Rev Bras Otorrinolaringol. 2004;70(6):739-41.

28. Mattiske JA, Oates JM, Greenwood KM. Voice problems among teachers: a review of prevalence, causes, prevention and treatment. J Voice. 1998;12(4):489-99.

29. Russell A, Oates J, Greenwood KM. Prevalence of voice problems in teachers. J Voice. 1998;12(4):467-79.

30. Lehto L, Rantala L, Vilkman E, Alku P, Bäckström T. Experiences of a short vocal training course for call-centre customer service advisors. Folia Phoniatr Logop. 2003;55(4):163-76.

Anexo 1. Questionário de saúde vocal

Instruções: Por favor, responda todas as questões

I - Dados de identificação

1. Data

2. Idade:

3. Gênero:

Feminino ( )

Protocolo $\mathrm{N}^{\circ}$

5. Escolaridade:

Analfabeto ( )

Ensino fundamental incompleto ( )

Ensino fundamental completo ( )

Ensino médio incompleto ( )

Ensino médio completo ( )

Ensino superior incompleto ( )

Ensino superior completo ( )

Pós-graduação ( )

II - Dados de hábitos vocais

6. Há quanto tempo canta na igreja?

1 ano () 2 anos () 3 anos () 4 anos () mais de 5 anos ()

7. Possui classificação vocal?

1. $\operatorname{Sim}($ ) 2. Não ( )

8. Em caso afirmativo, qual?

Soprano () Mezzo ( ) Contralto ()

Baixo () Tenor ( ) Barítono ()

9. Faz aquecimento vocal?

1. Sim ( ) 2. Não ( )

10. Percebeu alguma mudança negativa na voz após iniciar atividade de canto na igreja?

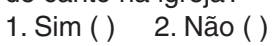

11. Quantos copos de água você ingere por dia?

1 a 2()$\quad 3$ a 4()$\quad 5$ a 6()$\quad$ mais de 7()
III - Queixas vocais

12. Percebe alguma das queixas abaixo na sua voz?

Rouquidão ( ) Dor no pescoço/nuca ( ) Garganta seca ( )

Pigarro constante ( ) Cansaço ao falar ( ) Ar na voz ( )

Perda de voz () Tosse constante ( ) Dor ao engolir ( )

Dor na garganta ao falar ( ) Voz fraca ( ) Falhas na voz ( )

Ardência na garganta ao falar ( )

Sensação de corpo estranho na garganta ( ) Nenhuma ( )

Outros:

IV - Dificuldades vocais

13. Percebe algum dos problemas abaixo quando canta ? (desconsiderar períodos relacionados a doenças ou período menstrual)

Dificuldade em alcançar notas agudas ( )

Dificuldade em realizar a transição de uma região média para aguda? ()

Rouquidão ( )

ar na voz ( )

falhas na voz( )

perda da voz - afonia ( )

Desconforto na garganta ( )

Dor na garganta ( )

Nenhuma()

$\mathrm{V}$ - Abusos vocais antes da atividade de canto

14. Você grita? Sim ( ) Não ( )

15. Fala "alto"? Sim () Não ( )

16. Fala excessivamente? Sim ( ) Não ( )

17. Faz uso de gelados Sim ( ) Não ( ) 